\title{
ON COLLECTIONWISE NORMALITY OF PRODUCT SPACES. I
}

\author{
KEIKO CHIBA
}

\begin{abstract}
In this paper the following result will be obtained: Let $X$ be a collectionwise normal $\Sigma$-space (in the sense of Nagami [9]) and $Y$ a paracompact first countable $P$-space (in the sense of Morita [8]). Then $X \times Y$ is collectionwise normal.
\end{abstract}

1. Introduction. Throughout this paper all spaces are Hausdorff spaces.

On collectionwise normality of a product space $X \times Y$, the following theorems are known.

(I) (KomBAROV [5]). Let $X$ be a normal countably compact space and $Y$ a paracompact sequential space. Then $X \times Y$ is collectionwise normal.

(II) (YAJIMA [14]). Let $X$ be a collectionwise normal space which has a $\sigma$ closure preserving closed cover by countably compact sets and $Y$ a paracompact first countable space. Then $X \times Y$ is collectionwise normal.

We shall consider another condition of $X$ and $Y$ such that $X \times Y$ is collectionwise normal. The following are known.

(III) (NAGAMI [9]). Let $X$ be a paracompact $\Sigma$-space and $Y$ a paracompact $P$-space. Then $X \times Y$ is paracompact.

(IV) [2]. Let $X$ be a normal $M$-space and $Y$ a paracompact first countable $P$-space. Then $X \times Y$ is normal.

(V) [2]. There exist a normal $\sigma$-space $X$ and a compact first countable space $Y$ such that $X \times Y$ is not normal (see $\S 3$, Example 3).

In this paper we shall prove the following theorem which contains (IV).

THEOREM. Let $X$ be a collectionwise normal $\Sigma$-space and $Y$ a paracompact first countable $P$-space. Then $X \times Y$ is collectionwise normal.

The definitions of $\Sigma$-spaces are due to Nagami [9], $P$-spaces and $M$-spaces are due to Morita [8], and $\sigma$-spaces are due to Okuyama [10].

2. Proof of Theorem. For the proof, we shall use the following facts.

FACT 1. Let $\mathfrak{A}=\left\{A_{\gamma} \mid \gamma \in \Gamma\right\}$ be a discrete collection of closed subsets of $X$. If there exists a normal open cover of $X$ each of whose members meets at most

Received by the editors June 20, 1983 and, in revised form, October 27, 1983. The author presented the contents of this paper at a summer seminar on July 27, 1982.

1980 Mathematics Subject Classification. Primary 54B10; Secondary 54D15.

Key words and phrases. Product space, normal, collectionwise normal, paracompact, $\Sigma$-space, $P$-space, first countable. 
one $A_{\gamma}$, then there are open sets $H_{\gamma}$ of $X$ such that $H_{\gamma} \supset A_{\gamma}$ for each $\gamma \in \Gamma$ and $H_{\gamma} \cap H_{\mu}=\emptyset$ if $\gamma \neq \mu$.

Fact 1 is well known.

FACT 2 [9, Lemma 1.4]. Let $X$ be a $\Sigma$-space. Then $X$ has a $\Sigma$-net $\left\{\mathfrak{F}_{n} \mid n=\right.$ $1,2, \ldots\}$ which satisfies the following conditions:

$\left(\mathrm{N}_{1}\right) \mathfrak{F}_{n}=\left\{F\left(\alpha_{1}, \ldots, \alpha_{n}\right) \mid \alpha_{1}, \ldots, \alpha_{n} \in \Xi\right\}$.

$\left(\mathrm{N}_{2}\right)$ Every $F\left(\alpha_{1}, \ldots, \alpha_{n}\right)=\bigcup\left\{F\left(\alpha_{1}, \ldots, \alpha_{n}, \alpha_{n+1}\right) \mid \alpha_{n+1} \in \Xi\right\}$.

$\left(\mathrm{N}_{3}\right)$ For every $x \in X$, there exists a sequence $\alpha_{1}, \alpha_{2}, \ldots$ such that $\left\{F\left(\alpha_{1}, \ldots, \alpha_{n}\right) \mid\right.$ $n=1,2, \ldots\}$ is a net of $C(x)$.

Here $C(x)=\bigcap\left\{C\left(x, \mathfrak{F}_{n}\right) \mid n=1,2, \ldots\right\}, C\left(x, \mathfrak{F}_{n}\right)=\bigcap\left\{F \mid x \in F \in \mathfrak{F}_{n}\right\}$.

Proof OF THEOREM. ${ }^{1}$ This proof is a modification of that of (III) (Theorem 4.1 in [9]). Let $X$ be a collectionwise normal $\Sigma$-space and $Y$ a paracompact first countable $P$-space. Let $\left\{\mathfrak{F}_{n} \mid n=1,2, \ldots\right\}$ be a $\Sigma$-net of $X$ satisfying the conditions $\left(\mathrm{N}_{1}\right)-\left(\mathrm{N}_{3}\right)$ in Fact 2 . Since $\mathfrak{\mho}_{n}$ is a locally finite closed cover of $X$ and $X$ is strongly normal, by Katětov [4], there exists a locally finite cozero-set cover $\mathfrak{H}_{n}=\left\{H\left(\alpha_{1}, \ldots, \alpha_{n}\right) \mid \alpha_{1}, \ldots, \alpha_{n} \in \Xi\right\}$ such that

$$
F\left(\alpha_{1}, \ldots, \alpha_{n}\right) \subset H\left(\alpha_{1}, \ldots, \alpha_{n}\right) \text { for each } \alpha_{1}, \ldots, \alpha_{n} \in \Xi \text {. }
$$

Let $\mathfrak{A}$ be a discrete family of closed subsets of $X \times Y$. Let $\mathfrak{W}\left(\alpha_{1}, \ldots, \alpha_{n}\right)=\left\{U_{\lambda} \times\right.$ $\left.V_{\lambda}(\neq \emptyset) \mid \lambda \in \Lambda\left(\alpha_{1}, \ldots, \alpha_{n}\right)\right\}$ be the maximal collection satisfying the following conditions:

(1) Each $U_{\lambda}$ is a finite union of cozero-sets $\left\{U_{\lambda, j} \mid 1 \leq j \leq m(\lambda)\right\}$ of $X$ such that

$$
F\left(\alpha_{1}, \ldots, \alpha_{n}\right) \subset U_{\lambda} \subset H\left(\alpha_{1}, \ldots, \alpha_{n}\right) .
$$

(2) Each $V_{\lambda}$ is an open set of $Y$.

(3) Each memeber of $\mathfrak{J}_{\lambda}=\left\{U_{\lambda, j} \times V_{\lambda} \mid 1 \leq j \leq m(\lambda)\right\}$ meets at most one member of $\mathfrak{A}$.

Let us put $V\left(\alpha_{1}, \ldots, \alpha_{n}\right)=\bigcup\left\{V_{\lambda} \mid \lambda \in \Lambda\left(\alpha_{1}, \ldots, \alpha_{n}\right)\right\}$. Then $V\left(\alpha_{1}, \ldots, \alpha_{n}\right) \subset$ $V\left(\alpha_{1}, \ldots, \alpha_{n}, \alpha_{n+1}\right)$ for each $\alpha_{1}, \ldots, \alpha_{n}, \alpha_{n+1} \in \Xi$. Since $Y$ is a $P$-space, for each $\alpha_{1}, \ldots, \alpha_{n} \in \Xi$, there exists a closed set $K\left(\alpha_{1}, \ldots, \alpha_{n}\right)$ of $Y$ such that

(4) $K\left(\alpha_{1}, \ldots, \alpha_{n}\right) \subset V\left(\alpha_{1}, \ldots, \alpha_{n}\right)$.

(5) If $\bigcup_{n=1}^{\omega} V\left(\alpha_{1}, \ldots, \alpha_{n}\right)=Y$, then $\bigcup_{n=1}^{\omega} K\left(\alpha_{1}, \ldots, \alpha_{n}\right)=Y$, where $\omega$ denotes the first infinite ordinal.

Since $Y$ is paracompact, for each $\alpha_{1}, \ldots, \alpha_{n} \in \Xi$, there exists a locally finite collection $\left\{V_{\lambda}^{\prime} \mid \lambda \in \Lambda\left(\alpha_{1}, \ldots, \alpha_{n}\right)\right\}$ of cozero-sets in $Y$ such that:

(6) $V_{\lambda}^{\prime} \subset V_{\lambda}$ for each $\lambda \in \Lambda\left(\alpha_{1}, \ldots, \alpha_{n}\right)$.

(7) $K\left(\alpha_{1}, \ldots, \alpha_{n}\right) \subset \bigcup\left\{V_{\lambda}^{\prime} \mid \lambda \in \Lambda\left(\alpha_{1}, \ldots, \alpha_{n}\right)\right\}$.

Let us put $\mathfrak{G}_{n}=\left\{U_{\lambda, j} \times V_{\lambda}^{\prime} \mid \lambda \in \Lambda\left(\alpha_{1}, \ldots, \alpha_{n}\right), \alpha_{1}, \ldots, \alpha_{n} \in \Xi, 1 \leq j \leq m(\lambda)\right\}$ for each $n=1,2, \ldots$, and put $\mathfrak{G}=\bigcup\left\{\mathfrak{G}_{n} \mid n=1,2, \ldots\right\}$.

Then we have:

(8) Each $\mathfrak{G}_{n}$ is locally finite in $X \times Y$.

(9) Each member of $\mathfrak{G}$ meets at most one member of $\mathfrak{A}$.

(10) Each member of $\mathfrak{G}$ is a cozero-set in $X \times Y$.

(11) $\mathfrak{G}$ is a cover of $X \times Y$.

(8)-(10) are clear.

\footnotetext{
${ }^{1}$ The author first proved this theorem by another method. Y. Yajima pointed out that we can give a simpler proof by modifying the proof of (III).
} 
ProOF OF (11). Let $(x, y) \in X \times Y$ be an arbitrary element. Let $\alpha_{1}, \ldots, \alpha_{n}, \ldots$ $\in \Xi$ be elements such that $\left\{F\left(\alpha_{1}, \ldots, \alpha_{n}\right) \mid n=1,2, \ldots\right\}$ is a net of $C(x)$. Then we have $\bigcup_{n=1}^{\omega} V\left(\alpha_{1}, \ldots, \alpha_{n}\right)=Y$. To show this, let $y^{\prime}$ be an arbitrary element of $Y$. Then, since $C(x)$ is countably compact [9] and the family $\mathfrak{A}$ is discrete, $\left\{A_{\xi} \in \mathfrak{A} \mid\left(C(x) \times\left\{y^{\prime}\right\}\right) \cap A_{\xi} \neq \emptyset\right\}$ is finite. Therefore, by using the first countability of $Y$, there is a finite family $\left\{M_{j} \mid j=1,2, \ldots, k\right\}$ of open sets in $X$ and an open set $G$ in $Y$ such that:

(12) $C(x) \subset \bigcup_{j=1}^{k} M_{j}, y^{\prime} \in G$.

(13) Each $M_{j} \times G$ meets at one member of $\mathfrak{A}$.

By Lemma 2.1 in [14], there are cozero-sets $M_{j}^{\prime}$ in $X$ such that $M_{j}^{\prime} \subset M_{j}$ and $C(x) \subset$ $\bigcup_{j=1}^{k} M_{j}^{\prime}$. Then $F\left(\alpha_{1}, \ldots, \alpha_{i}\right) \subset \bigcup_{j=1}^{k} M_{j}^{\prime}$ for some $i$. Let us put $U_{j}=M_{j}^{\prime} \cap$ $H\left(\alpha_{1}, \ldots, \alpha_{i}\right)$. Then $U_{j}$ are cozero-sets in $X$ and $\left(\bigcup_{j=1}^{k} U_{j}\right) \times G \in \mathfrak{W}\left(\alpha_{1}, \ldots, \alpha_{i}\right)$ by the maximality of $\mathfrak{W}\left(\alpha_{1}, \ldots, \alpha_{i}\right)$. Thus $y^{\prime} \in V\left(\alpha_{1}, \ldots, \alpha_{i}\right)$.

Therefore we have $\bigcup_{n=1}^{\omega} K\left(\alpha_{1}, \ldots, \alpha_{n}\right)=Y$ by (5). Hence $y \in K\left(\alpha_{1}, \ldots, \alpha_{n}\right)$ for some $n$. By (7), $y \in V_{\lambda}^{\prime}$ for some $\lambda \in \Lambda\left(\alpha_{1}, \ldots, \alpha_{n}\right)$. Then

$$
(x, y) \in C(x) \times\{y\} \subset F\left(\alpha_{1}, \ldots, \alpha_{n}\right) \times V_{\lambda}^{\prime} \subset U_{\lambda} \times V_{\lambda}^{\prime} .
$$

Since $x \in U_{\lambda, j}$ for some $j \leq m(\lambda),(x, y) \in U_{\lambda, j} \times V_{\lambda}^{\prime} \in \mathfrak{G}_{n} \subset \mathfrak{G}$.

By (8)-(11), $\mathfrak{G}$ is a normal open cover of $X \times Y$, each of whose members meets at most one element of $\mathfrak{A}$. By Fact 1 , there exists a disjoint family $\left\{H_{A} \mid A \in \mathfrak{A}\right\}$ of open sets in $X \times Y$ such that $H_{A} \supset A$ for each $A \in \mathfrak{A}$. Hence $X \times Y$ is collectionwise normal. The proof of the Theorem is complete.

\section{Remarks and examples.}

REMARK 1. Our Theorem is neither contained in (I) nor (II) in $\S 1$. In fact, let $X$ be the space of irrationals of $R$ with the euclidean topology where $R$ is the real line and $Y$ the Michael line [6]; then $Y$ is a paracompact first countable space and $X \times Y$ is not normal [6]. Therefore $X$ does not satisfy the condition in (II). Also $X$ is not countably compact. But $X$ is a collectionwise normal $\Sigma$-space.

Moreover, this example shows that the condition "Yis a $P$-space" cannot be dropped in the Theorem

REMARK 2. We cannot weaken the condition " $Y$ is first countable" to the condition "for each $y \in Y$ is a $G_{\delta}$-set". In fact the following example exists.

EXAMPLE $1[2]$. Let $X=\left[0, \omega_{1}\right)=\left\{\alpha \mid \alpha<\omega_{1}\right\}$ with the order topology where $\omega_{1}$ is the first uncountable ordinal. Then it is well known that $X$ is a normal countably compact space. Let $Y=\left([0, \omega) \times\left[0, \omega_{1}\right)\right) \cup\left\{\left(\omega, \omega_{1}\right)\right\}$ with the topology as follows: $\left\{\left([\alpha, \omega] \times\left[\beta, \omega_{1}\right]\right) \cap Y \mid \alpha<\omega, \beta<\omega_{1}\right\}$ is a neighborhood base of $\left(\omega, \omega_{1}\right)$ and for each $y \in Y-\left\{\left(\omega, \omega_{1}\right)\right\}, y$ is an isolated point of $Y$. Then $Y$ is a paracompact perfectly normal space but $Y$ is not first countable. Also $X \times Y$ is not normal [2].

REMARK 3. The paracompactness of $Y$ cannot be weakened to the condition "collectionwise normal". In fact the following example exists.

EXAMPLE 2. There exists a compact space $X$ and a collectionwise normal perfectly normal first countable space $Y$ such that $X \times Y$ is not normal. Let $Y$ be the space constructed by R. Pol in [11]. Then $Y$ has the above properties, but $Y$ is not paracompact. Therefore, by the Theorem of Tamano [13], there exists a compact space $X$ such that $X \times Y$ is not normal. 
REMARK 4. The condition " $X$ is a $\Sigma$-space" cannot be replaced by the condition " $X$ is a $P$-space". In fact, let $X$ be the Sorgenfrey line $[\mathbf{1 2}]$; then $X$ is a paracompact first countable $P$-space such that $X^{2}=X \times X$ is not normal.

EXAMPLE 3 [2]. There exists a normal $\sigma$-space $X$ and a compact first countable space $Y$ such that $X \times Y$ is not normal. Let $Y$ be the "two arrow space" i.e., let $E$ be the unit square with lexicographic order (cf. [7, Example 10.4]). Let $Y=(\{y \mid 0<y \leq 1\} \times\{0\}) \cup(\{y \mid 0 \leq y<1\} \times\{1\})$ with the subspace topology of $E$. Then $Y$ is a compact first countable space. Let $X$ be the space in Bing's Example $\mathrm{H}[\mathbf{1}]$ constructed by a suitable set $P$. Then $X$ is a normal $\sigma$-space and $X \times Y$ is not normal (this follows from the proof of Theorem 1 in [3] because $Y$ is separable and not metrizable; also see p. 6 in [2]).

REMARK 5. This author does not know whether we can generalize the condition " $Y$ is first countable" to " $Y$ is sequential" or not.

\section{REFERENCES}

1. R. H. Bing, Metrization of topological spaces, Canad. J. Math. 3 (1951), 175-186.

2. K. Chiba, On products of normal spaces, Rep. Fac. Sci. Shizuoka Univ. 9 (1974), 1-11.

3. T. Chiba and K. Chiba, A note on normality of product spaces, Sci. Rep. Tokyo Kyoiku Daigaku 12 (1974), 55-63.

4. M. Katětov, On expansion of locally finite coverings, Colloq. Math. 6 (1958), 145-151.

5. A. P. Kombarov, On the product of normal spaces. Uniformities on $\Sigma$-products, Soviet Math. Dokl. 13 (1972), 1068-1071.

6. E. Michael, The product of a normal space and a metric space need not be normal, Bull. Amer. Math. Soc. 69 (1963), 375-376.

7.

8. K. Morita, Products of normal spaces with metric spaces, Math. Ann. 154 (1964), 365-382.

9. K. Nagami, $\Sigma$-spaces, Fund. Math. 65 (1969), 169-192.

10. A. Okuyama, Some generalizations of metric spaces, their metrization theorems and product spaces, Sci. Rep. Tokyo Kyoiku Daigaku 9 (1967), 236-254.

11. R. Pol, A perfectly normal locally metrizable non-paracompact space, Fund. Math. 97 (1977), 37-42.

12. R. H. Sorgenfrey, On the topological product of paracompact spaces, Bull. Amer. Math. Soc. 53 (1947), 631-632.

13. H. Tamano, On compactifications, J. Math. Kyoto Univ. 1-2 (1962), 162-193.

14. Y. Yajima, Topological games and products. I, Fund. Math. 113 (1981), 141-153.

Department of Mathematics, FaCulty of Science, Shizuoka University, OHYA, SHIZUOKA 422 , JAPAN 